\title{
Dégradation dans le rumen de l'azote des parois d'une même luzerne, verte ou ensilée
}

\author{
J Aufrère, D Boulberhane, D Graviou \\ INRA, station de recherche sur la nutrition des herbivores, Theix, \\ 63122 Saint-Genès-Champanelle, France
}

L'azote (N) des fourrages lié à la fraction NDF (NNDF) est constitué essentiellement de l' $N$ des protéines dénaturées par la chaleur ou liées aux tannins, et de l'N lié à la fraction ADF (NADF). notamment de I'N de l'extensine, glycoprotéine de la paroi primaire riche en hydroxyproline (Lamport, 1967). Le NNDF est considéré comme étant en partie dégradé dans le rumen tandis que le NADF est considéré comme non dégradé (Van Soest, 1982). Notre objectif est de vérifier cette dernière hypothèse.

Nous avons mesuré par la technique des sachets de nylon, chez 4 moutons, la dégradation dans le rumen de I'N, d'une luzerne verte (FV) et de 2 ensilages préparés à partir de ce même fourrage, l'un sans conservateur (E1), et l'autre avec addition de 5 Vhonne d'acide formique à $80 \%$ (E2). Les animaux recevaient les mêmes fourrages que ceux introduits dans les sachets. Aprés $2,4,8,16,24$ et $48 \mathrm{~h}$ d'incubation, les résidus de sachets ont été décontaminés au stomacher et séchés à $60^{\circ}$. Sur les fourrages et sur les résidus de sachets ont été déterminées les teneurs en azote total (Nt), NDF, ADF, NNDF, NADF, ainsi que la teneur en hydroxyproline du NDF et de l'ADF des fourrages, et de l'ADF des résidus des sachets après $16 \mathrm{~h}$ et $48 \mathrm{~h}$ de séjour dans le rumen.

Pour des fourrages ayant des teneurs en Nt très proches (respectivement de $2,85 \%, 2,78 \%$ et $3,03 \%$ de la matière sèche pour FV, E1, E2), la dégradabilité théorique (DT) de I' $\mathrm{N}$ a été de $84,7 \%$ pour $F V, 85,2 \%$ pour $E 1$ et $89,3 \%$ pour $E 2$. L'N des parois représente une part trop faible de l' $N$ total (moins de $11 \%$ pour celui du NDF et moins de $7 \%$ pour celui de l'ADF) des fourrages étudiés pour qu'il puisse avoir une incidence importante sur la DT. Cependant nous avons observé pour ces 3 fourrages une dégradabilité de $40,9 \%$ (FV), 22\% (E1), 39\% (E2) non seulement pour le NNDF, mais également de $37,4 \%$ (FV), $28 \%$ (E1), 15\% (E2) pour le NADF.

La disparition partielle de l'hydroxyproline lié à I'ADF dans les résidus (tableau I) indique que l'extensine est dégradable dans le rumen. Cela peut expliquer en partie la dégradation du NADF observée pour ces fourrages.

Lamport DTA (1967) Nature 216, 1322-1324

Van Soest PJ (1982) in : Nutritional Ecology of the Ruminant (PJ Van Soest, ed) 118-138

Tableau I. Teneurs en azote de l'ADF (NADF) et en azote de l'hydroxyproline de l'ADF (N OH proline) (exprimées par rapport à Nt de l'aliment) dans les fourrages (fourrage vert et ensilages de luzerne) et dans les résidus de sachets aux temps 16 et $48 \mathrm{~h}$.

NADFNt (\%)

NOH Proline de l'ADF/Nt (\%)

Aliments Résidu $16 \mathrm{~h}$ Résidu $48 \mathrm{~h}$

Aliment Résidu $16 \mathrm{~h}$ Résidu $48 \mathrm{~h}$

$\begin{array}{lllllll}\text { Fourrage vert de luzerne } & 6,27 & 3,05 & 2,68 & 0,31 & 0,10 & 0,09 \\ \text { Ensilage sans conservateur } & 7,33 & 4,52 & 3,03 & 0,43 & 0,17 & 0,15 \\ \text { Ensilage + acide formique } & 5,91 & 3,03 & 2,99 & 0,35 & 0,12 & 0,09\end{array}$

Fourrage vert de luzerne

Ensilage + acide formique
7,33

3,03

2,99
0,35

0,12

0,09 